
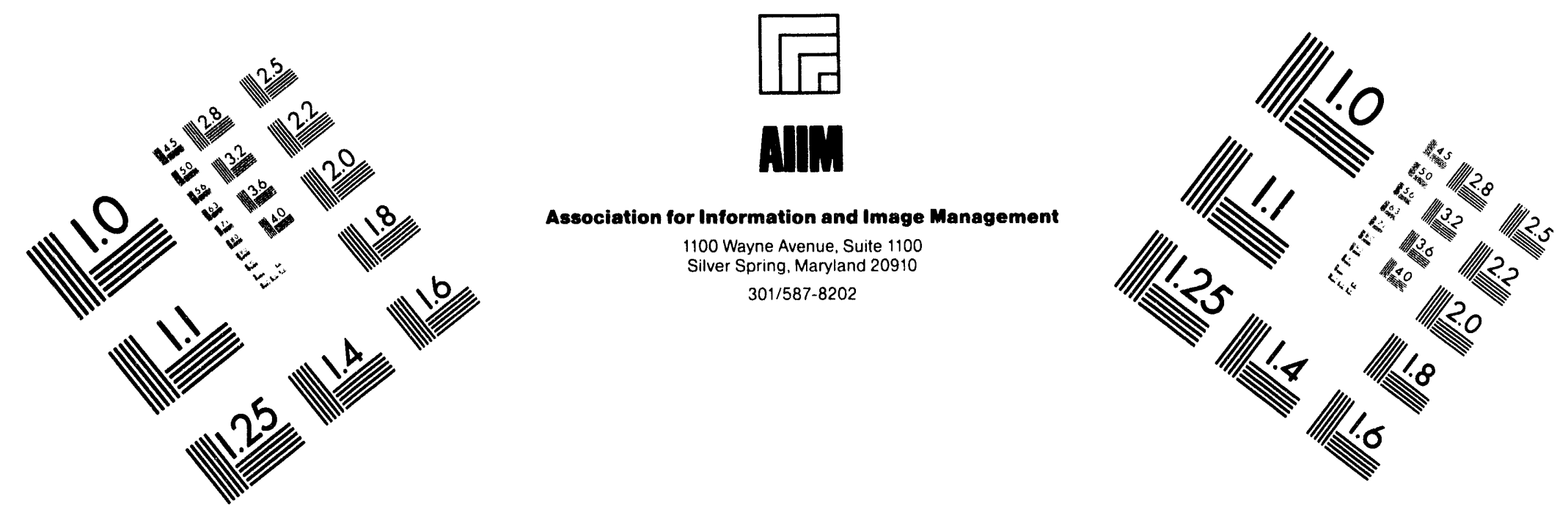

\title{
Centimeter
}

$\begin{array}{llllllllllllllll}1 & 2 & 3 & 4 & 5 & 6 & 7 & 8 & 9 & 10 & 11 & 12 & 13 & 14 & 15 & \mathrm{~mm}\end{array}$

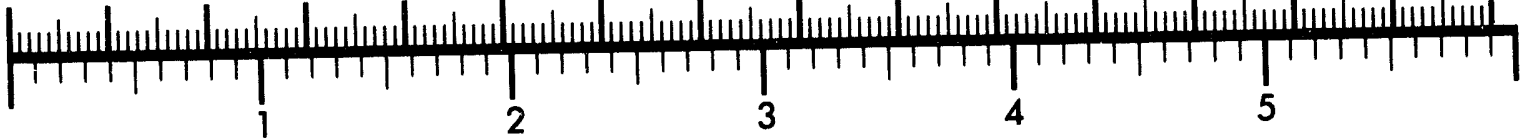
Inches
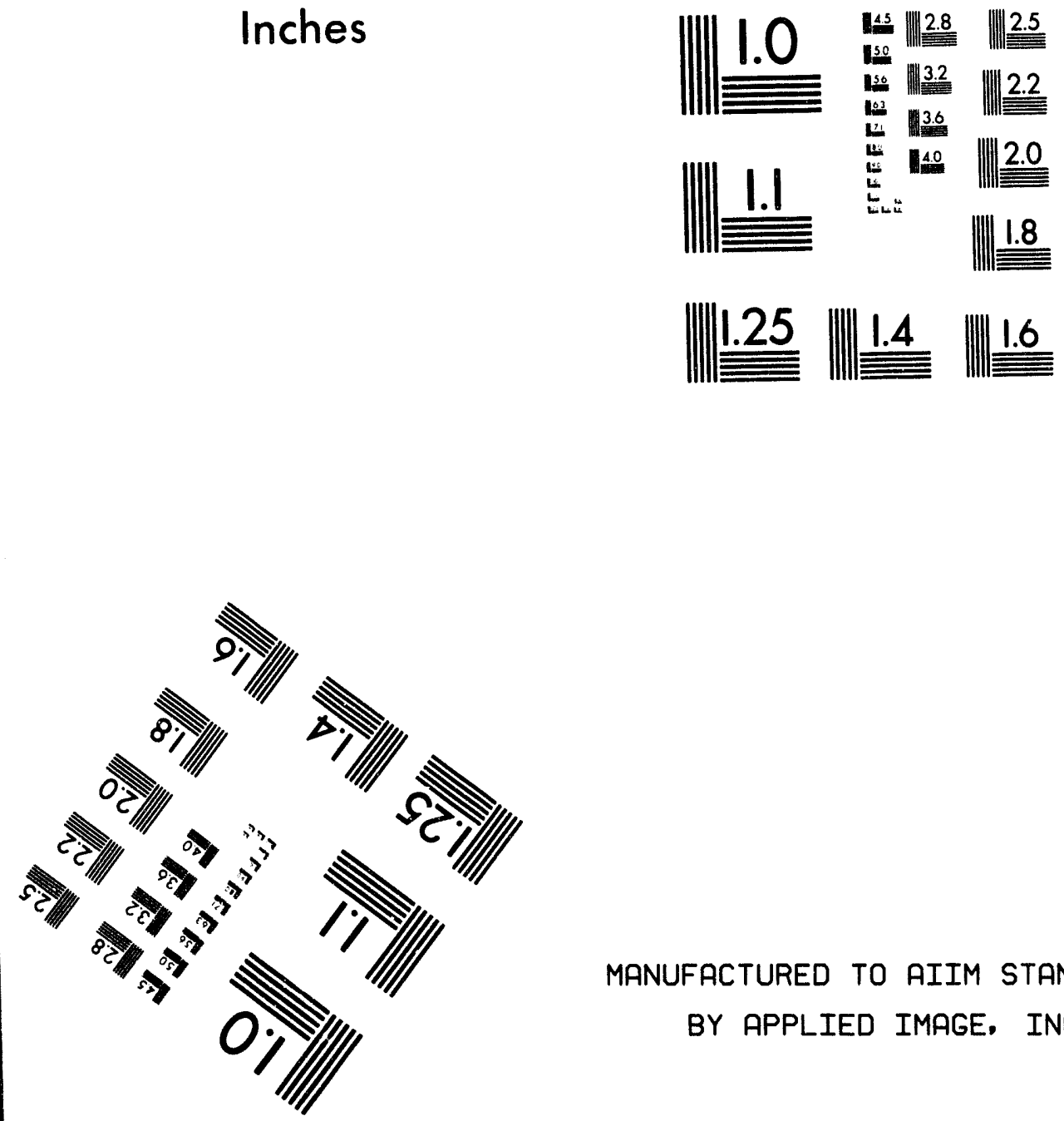

MANUFACTURED TO AIIM STANDARDS BY APPLIED IMAGE, INC.

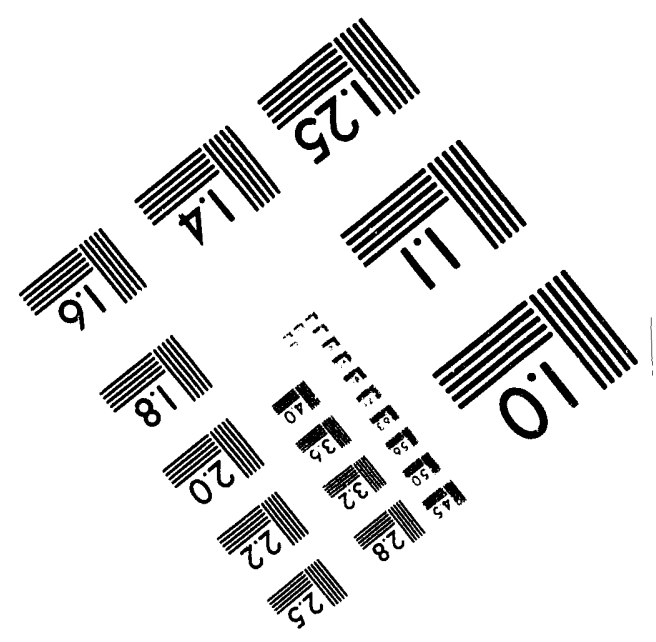



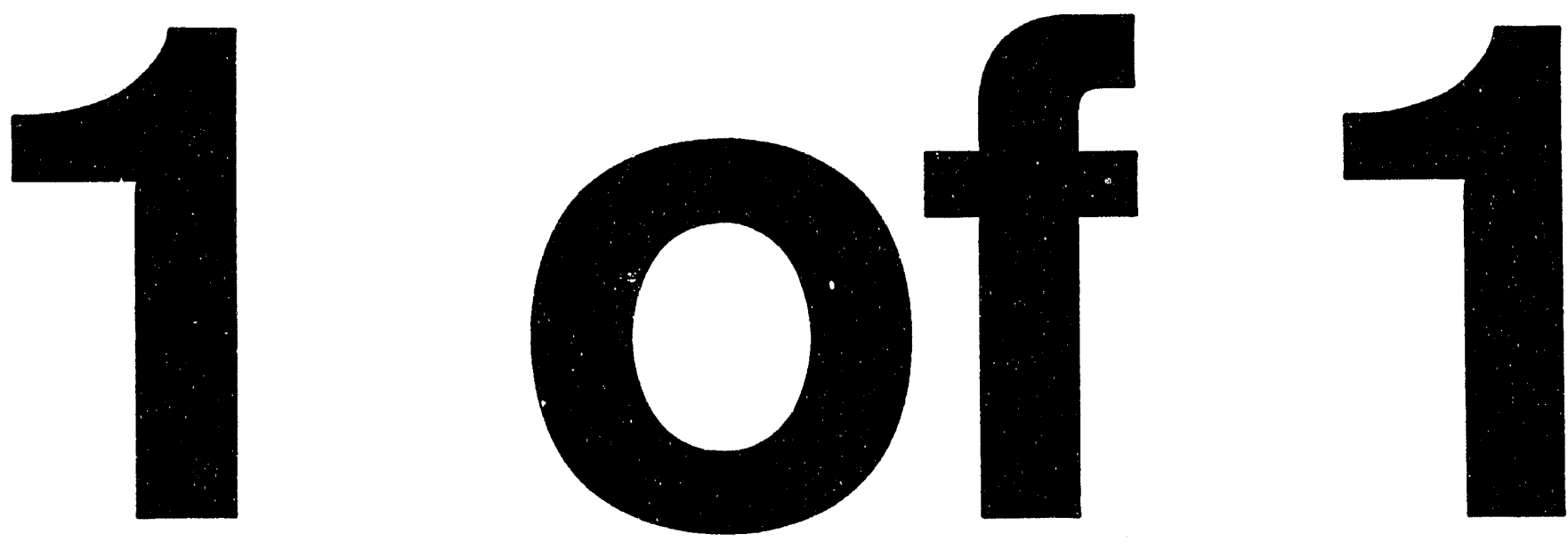


\title{
ANK/XFD/CP-82879 \\ Conf-940714--12
}

\section{Phase Errors and Predicted Spectral Performance of a Prototype Undulator}

\author{
Reger J.Dejus, Isaac Vasserman, Elizabeth R. Moog, and Efim Gluskin \\ Advanced Photon Source \\ Argonne National Laboratory \\ 9700 S. Cass Ave \\ Argonne, IL 60439
}

A prototype undulator ( $3.3 \mathrm{~cm}$-period, permanent-magnet hybrid device) has been used to study different magnetic end-configurations and shimming techniques for straightening the beam trajectory. Field distributions obtained by Hall probe measurements were analyzed in terms of trajectory, phase errors, and on-axis brightness for the purpose of correlating predicted spectral intensity with the calculated phase errors. Two device configurations were analyzed. One configuration had a full-strength first magnet at each end and the next-to-last pole was recessed to make the trajectory through the middle of the undulator parallel to the undulator axis. For the second configuration, the first permanent magnet at each end was replaced by a half-strength magnet to reduce the trajectory displacement and the next-to-last pole was adjusted appropriately, and shims were added to straighten the trajectory. Random magnetic field errors can cause trajectory deviations that will affect the optimum angle for viewing the emitted radiation, and care must be taken to select the appropriate angle when calculating the phase errors. This angle may be calculated from the average trajectory angle evaluated at the location of the poles. For the second configuration, we find an rms phase error of less than $3^{\circ}$ and predict $8 \% \%$ of the ideal value of the on-axis brightness for the third harmonic (nominal gap is $11.5 \mathrm{~mm}$ and derived effective $K$ is 2.25 ). We have also analyzed the gap dependence of the phase errors and spectral brightness and have found that the rms phase error remain small at all gap settings.

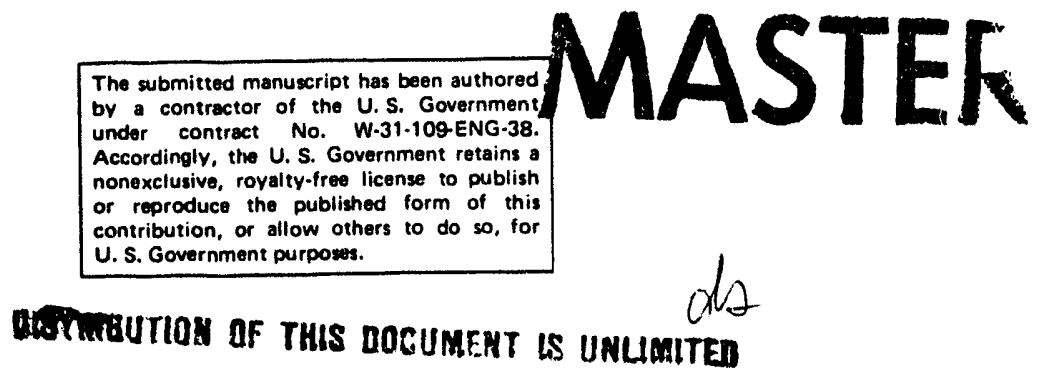




\section{INTRODUCTION}

The spectral performance of a real undulator may be examined by studying the radiated intensity of the odd harmonics derived from a measured magnetic field map. Random magnetic field errors and other structural defects will be detrimental to the peak intensity of the harmonics (with the higher harmonics being more reduced than the lower), and the spectral quality of an undulator may therefore be expressed in terms of how well it performs in relation to the estimated performance of an ideal device.

In the past, the most common figure of merit for predicting the reduction of the on-axis brightness has been the rms peak magnetic field error. However, it has been shown that this figure of merit is not strongly correlated with the observed reduction in peak intensity, and the rms phase error has instead been suggested as a better figure of merit. ${ }^{1}$ In this work, we present the calculated phase errors and the predicted on-axis brightness for a prototype undulator used at the Advanced Photon Source for studying magnetic end-configurations and shimming techniques. Peak intensity reductions will be discussed in relation to the calculated phase errors and comparisons will be made with the model proposed by Walker. 2

\section{UNDULATOR MAGNETIC CONFIGURATIONS}

The undulator is a permanent-magnet, hybrid device with a period length of 3.3 $\mathrm{cm}$ and 24 full-strength magnetic periods. The magnet material is $\mathrm{Nd}-\mathrm{Fe}-\mathrm{B}$ and the device has wedge-shaped pole pieces of vanadium-permendur for increased on-axis magnetic field. The undulator gap is adjustable with a minimum setting of $10.5 \mathrm{~mm}$ with a $B_{\text {eff }}$ of 0.84 Tesla $(K=2.59)$.

Two device configurations were analyzed at a nominal gap of $11.5 \mathrm{~mm}$ : configuration FS had a full-strength first magnet at each end and the next-to-last pole was recessed to make the trajectory through the middle of the undulator parallel to the 
undulator axis, and configuration HS had the first permanent magnet at each end replaced by a half-strength magnet to reduce the trajectory displacement and the next-to-last pole was adjusted appropriately. For configuration HS, shims were added to further straighten the trajectory. The gap dependence of the phase errors and the spectral brightness was also analyzed for the HS configuration. The measured magnetic profiles obtained by Hall probe measurements at a gap of $11.5 \mathrm{~mm}$ for the two configurations are shown in Figure 1.

\section{PHASE ERRORS AND PREDICTED SPECTRAL PERFORMANCE}

The trajectory, the radiation phase, and the on-axis brightness are calculated directly from the measured magnetic fields. The trajectory is obtained from the equations of motion, and the important phase-function $\delta(z)$ is calculated from

$\delta(z)=\omega\left(t^{\prime}-\frac{z}{c}\right)=\frac{2 \pi}{\lambda} \int_{0}^{z} \frac{1-\beta_{z}\left(z^{\prime}\right)}{\beta_{z}\left(z^{\prime}\right)} d z^{\prime}$,

where $c$ is the speed of light, and $\beta_{z} c$ is the electron velocity along the undulator ( $z$-axis). The radiation phase varies slowly near the poles and rapidly between them for large $\mathrm{K}$, and the behavior near the poles is important for maintaining high correlation between photons emitted at different poles. For an ideal device, the phase shows a perfect linear variation with position $z$ when evaluated only at the poles. For a real device, random or systematic deviations will substantially reduce the on-axis brightness. We define therefore the phase errors as the deviations from a linear fit to the phases calculated at the poles in the central periodic part of the undulator at the fundamental wavelength $\lambda=\lambda_{1}(0)$. The on-axis brightness is calculated using a general radiation formula in the far-field limit. ${ }^{3}$

In the analysis, we also make a least squares fit to the measured magnetic field data over the central periodic region for a determination of the Fourier components of the 
magnetic field, the effective field, and the period length. The fitting function consists of a sum of odd cosine functions

$$
B^{f i t}=\sum_{n=0} b_{2 n+1} \cos \left(\frac{2 \pi(2 n+1)}{\lambda_{0}}\left(z-z_{2 n+1}\right)\right),
$$

with the amplitudes $b_{n}$, the period length $\lambda_{0}$, and the phases $z_{n}$ as the fitting parameters.

The effective field is defined by

$$
B_{\text {eff }}=\sqrt{\sum_{n=0}\left(\frac{b_{2 n+1}}{2 n+1}\right)^{2}}
$$

and is used for calculating the performance of an ideal device.

\section{Results at 11.5-mm gap}

Random magnetic field errors can cause trajectory deviations that will affect the optimum angle for viewing the emitted radiation, and care must be taken to select the appropriate angle when calculating the phase errors. This angle may be calculated from the average trajectory angle evaluated at the location of the poles. The trajectory at 7.0 $\mathrm{GeV}$ for the HS configuration viewed at an optimized view angle of $2.3 \mu \mathrm{rad}$ is shown in Figure 2 (a) with the corresponding phase errors shown in Figure 2 (b). The trajectory is well behaved with a small kink at $z=0$, where shims were added to straighten the trajectory. The rms phase error of $2.9^{\circ}$ is small, and a good spectral performance is expected.

The results for the FS configuration are significantly different as is seen in Figures 3 (a) and 3 (b). The calculated phase error $\left(6.6^{\circ}\right)$ suggests a rather poor spertral performance, and this is indeed observed. The rms phase erro: depends strongly on the number of end poles omitted in the analysis. It is obvious that the rms phase error will be reduced if the first and the last data points are excluded from the analysis [see Figure 3 (b)]. For such a case, the rms phase error would be only $4.3^{\circ}$. 
Figures 4 (a) and (b) show the predicted on-axis brightness of the third harmonic compared with the ideal performance for the two configurations. The analyses of the two configurations are summarized in Table $I$.

Gap dependence

The gap dependence of the phase errors and the spectral brightness is listed in Table II. The HS configuration was used for this investigation. The device was initially tuned at a gap of $11.5 \mathrm{~mm}$, and data were taken at different gaps with no further adjustments of the ends or added shims. For small gaps, the rms peak field error is less than $0.4 \%$ and the rms phase error is about $3^{\circ}$ or better; the predicted on-axis brightness of the third harmonic is in the upper $80 \%$ range of the ideal.

\section{DISCUSSION}

For a real insertion device with random magnetic field errors, the trajectory walkoff and the steering of the beam may be large if the device is not properly tuned. For a well-tuned device (by shimming or other techniques), there should be an almost straight average trajectory with a overall small trajectory deviation. The average trajectory needs to be straight to keep the phase errors small.

If the trajectory is observed at an angle, there will be a systematic difference in phase between the odd and even poles, which is easily seen in the phase error plot as a systematic variation in the phase, and this in turn is a signature of the introduction of even harmonics in the radiated spectrum. It is therefore important to properly adjust the view angle even though the derived angles are relatively small in the cases presented here. The magnitude of the introduced phase error (in degrees) due to angular error $\theta$ (radians) is 


$$
p=\left[\frac{\sin ^{-1}(\gamma \theta / K)}{\pi}+\frac{1}{2 \gamma^{2}}\left(\frac{3 K \gamma \theta}{2 \pi}\right) \sqrt{1-(\gamma \theta / K)^{2}}\left(\frac{\lambda_{0}}{\lambda_{1}}\right)\right] \times 360,
$$

and is typically a few degrees/ $\mu$ rad for the $K$ values and $\gamma$ used in this work.

The calculated rms phase error is sensitive to the chosen range of the good field region: for a device with poorly tuned magnetic ends, the phase errors are substantially reduced by excluding additional poles at the ends in the analysis, and a meaningful comparison cannot be made with the model proposed by Walker ${ }^{2}$ for reduction in peak intensity of the odd harmonics. For a tuned device (HS configuration), our calculations are in reasonably good agreement with the model. The studied undulator has relative few periods $(\mathrm{N}=24)$, which makes the treatment of the ends of the undulator important. For a meaningful comparison with the Walker model, the rms phase error and the on-axis brightness should both be calculated over the same length of the device.

There are other methods for calculating the phase errors, but we have found that the method used in this work (by subtracting a linear variation from the calculated phase) is the most robust. The program $m a^{4}$ was written specifically for fast evaluation of the spectral performance of insertion devices (10 s), and will be used in routine analyses of insertion devices at the magnetic measurement facility at the Advanced Photon Source.

The experience gained with this device indicates that insertion devices with small rms phase errors $\left(<3^{\circ}\right)$ can be fabricated, and our calculations predict that an on-axis brightness of almost $90 \%$ of the ideal of the third harmonic and better then $80 \%$ of the fifth harmonic may be obtained. The calculations indicate also that the same relative spectral intensity is expected when the emittance of the stored beam at the Advanced Photon Source is taken into account. 


\section{ACKNOWLEDGMENTS}

The authors wish to thank the computer support staff at the Advanced Photon

Source for their maintenance of computers and software. This work was supported by the

U.S. Department of Energy, BES-Materials Sciences, under contract No. W-31-109-

ENG-38.

\section{DISCLAIMER}

This report was prepared as an account of work sponsored by an agency of the United States Government. Neither the United States Government nor any agency thereof, nor any of their employees, makes any warranty, express or implied, or assumes any legal liability or responsibility for the accuracy, completeness, or usefulness of any information, apparatus, product, or bility for the accuracy, complets that its use would not infringe privately owned rights. Reference herein to any specific commercial product, process, or service by trade name, trademark, manufacturer, or otherwise does not necessarily constitute or imply its endorsement, recommendation, or favoring by the United States Government or any agency thereof. The views mendation, or favoring by the United States Government or any ate or reflect those of the United States Government or any agency thereof. 


\section{EIGURE CAPTIONS}

Figure 1 (a). Measured magnetic field for the HS configuration: insertion device with half-strength magnets at the ends and shims at the center. (b). Measured magnetic field for the FS configuration: insertion device with full-strength magnets at the ends.

Figure 2 (a). Calculated trajectory in the horizontal plane for a storage ring energy of 7.0 $\mathrm{GeV}$ for the HS configuration. The smooth solid curve indicates the average trajectory. (b). Derived phase errors for the same configuration. The shim added at the center of the device gives rise to a relative large phase shift at $z=0$. The rms phase error is $2.9^{\circ}$.

Figure 3 (a). Calculated trajectory in the horizontal plane for a storage ring energy of 7.0 $\mathrm{GeV}$ for the FS configuration. (b). Derived phase errors for the same configuration. The rms phase error is $6.6^{\circ}$. The rms phase error would be substantially smaller if the good field region (central periodic region) is made smaller. If one additional pole at each end of the device is omitted in the analysis, the rms field error is reduced to $4.3^{\circ}$.

Figure 4 (a). On-axis brightness of the third harmonic for the HS configuration (solid) in comparison with ideal performance for a 24-period device (dotted). The peak intensity is $87 \%$ of the ideal at $11.96 \mathrm{keV}$. (b). On-axis brightness of the third harmonic for the FS configuration (solid) in comparison with ideal performance for a 24-period device (dotted). The peak intensity is $66 \%$ of the ideal at $11.90 \mathrm{keV}$. 


\section{TABLES}

Table I. Magnetic analysis of the HS (half-strength end magnets) and FS (full strength end magnets) configurations at a gap of $11.5 \mathrm{~mm}$.

\begin{tabular}{|c|c|c|}
\hline$B_{\text {eff }}$ (Tesla) & $\begin{array}{l}\mathrm{HS} \\
0.7311 \\
2.253\end{array}$ & $\begin{array}{l}\text { FS } \\
0.7342\end{array}$ \\
\hline $\begin{array}{l}\mathrm{b} 3 / \mathrm{b} 1(\%) \\
\mathrm{b} 5 / \mathrm{b} 1(\%)\end{array}$ & $\begin{array}{l}1.15 \\
0.23 \\
2.3\end{array}$ & $\begin{array}{l}2.202 \\
1.19 \\
0.26 \\
1.9\end{array}$ \\
\hline view angle $(\mu \mathrm{rad})$ & & \\
\hline $\begin{array}{l}\left.\text { rms peak field error }(\%)^{2}\right) \\
\text { rms phase error } 2 \text { ) } \\
3^{\text {rd }} \text { harmonic intensity }(\%)\end{array}$ & $\begin{array}{l}0.49 \\
2.9^{\circ} \\
87\end{array}$ & $\begin{array}{l}1.05 \\
6.6^{\circ} \\
66\end{array}$ \\
\hline
\end{tabular}

1) $K=0.934 \lambda_{0}(\mathrm{~cm}) B_{e f f}(T) . B_{e f f}$ and $\lambda_{0}$ determined from the LSQ-fit. $\lambda_{0}=3.3000 \mathrm{~cm}$.

2) Values are for two end poles omitted at each end of the device. Values depend strongly on the number of end poles omitted in the analysis for the FS configuration. For the FS configuration: analysis with one end pole omitted gives a $2.27 \% \mathrm{rms}$ peak field error and a $15.0^{\circ} \mathrm{rms}$ phase errors, and with three endpoles omitted a $0.60 \%$ rms peak field error and a $4.3^{\circ} \mathrm{ms}$ phase error.

Table II. Gap dependence of important parameters for a tuned device.

\begin{tabular}{|c|c|c|c|c|}
\hline $\begin{array}{l}B_{\text {eff }} \text { (Tesla) } \\
\mathrm{K} \\
\text { b3/b1 (\%) } \\
\text { b5/b1 (\%) } \\
\text { view angle }\end{array}$ & $\begin{array}{l}10.5 \mathrm{~mm} \\
0.8406 \\
2.590 \\
1.73 \\
0.52 \\
0.4\end{array}$ & $\begin{array}{l}11.5 \mathrm{~mm} \\
0.7364 \\
2.269 \\
1.17 \\
0.24 \\
-0.8\end{array}$ & $\begin{array}{l}20.0 \mathrm{~mm} \\
0.2906 \\
0.895 \\
0.14 \\
0.01 \\
-1.4\end{array}$ & $\begin{array}{l}\frac{40.0 \mathrm{~mm}}{0.0428} \\
0.132 \\
0.02 \\
0.01 \\
-2.3\end{array}$ \\
\hline $\begin{array}{c}(\mu \mathrm{rad}) \\
\text { rms peak field }\end{array}$ & 0.35 & 0.33 & 0.86 & 1.27 \\
\hline $\begin{array}{l}\text { ertor (\%) } \\
\text { rms phase error } \\
3^{\text {rd }} \text { harmonic } \\
\text { intensity (\%) }\end{array}$ & $\begin{array}{l}3.2^{\circ} \\
85\end{array}$ & $\begin{array}{l}2.6^{\circ} \\
89\end{array}$ & $\begin{array}{l}2.2^{\circ} \\
85\end{array}$ & $\begin{array}{l}* \\
79\end{array}$ \\
\hline
\end{tabular}

* A reliable phase error could not be determined because the angular deviations at the nominal positi $\cdot 1$ of the poles were too large. 


\section{REEERENCES}

1 B.L. Bobbs, G̣. Rakowsky, P. Kennedy, R.A. Cover and D. Slater, Nucl. Instr. and Meth. A296 (1990) 574.

2 R.P. Walker, Nucl. Instr. and Meth. A335 (1993) 328.

3 J.D. Jackson, Classical Electrodynamics, 2nd Edition, John Wiley \& Sons, Inc. , New York (1975) p 668.

4 Program ma (for magnetic analysis), Roger J. Dejus, unpublished. Code was written in the IDL programming language and may be obtained by contacting the author. 


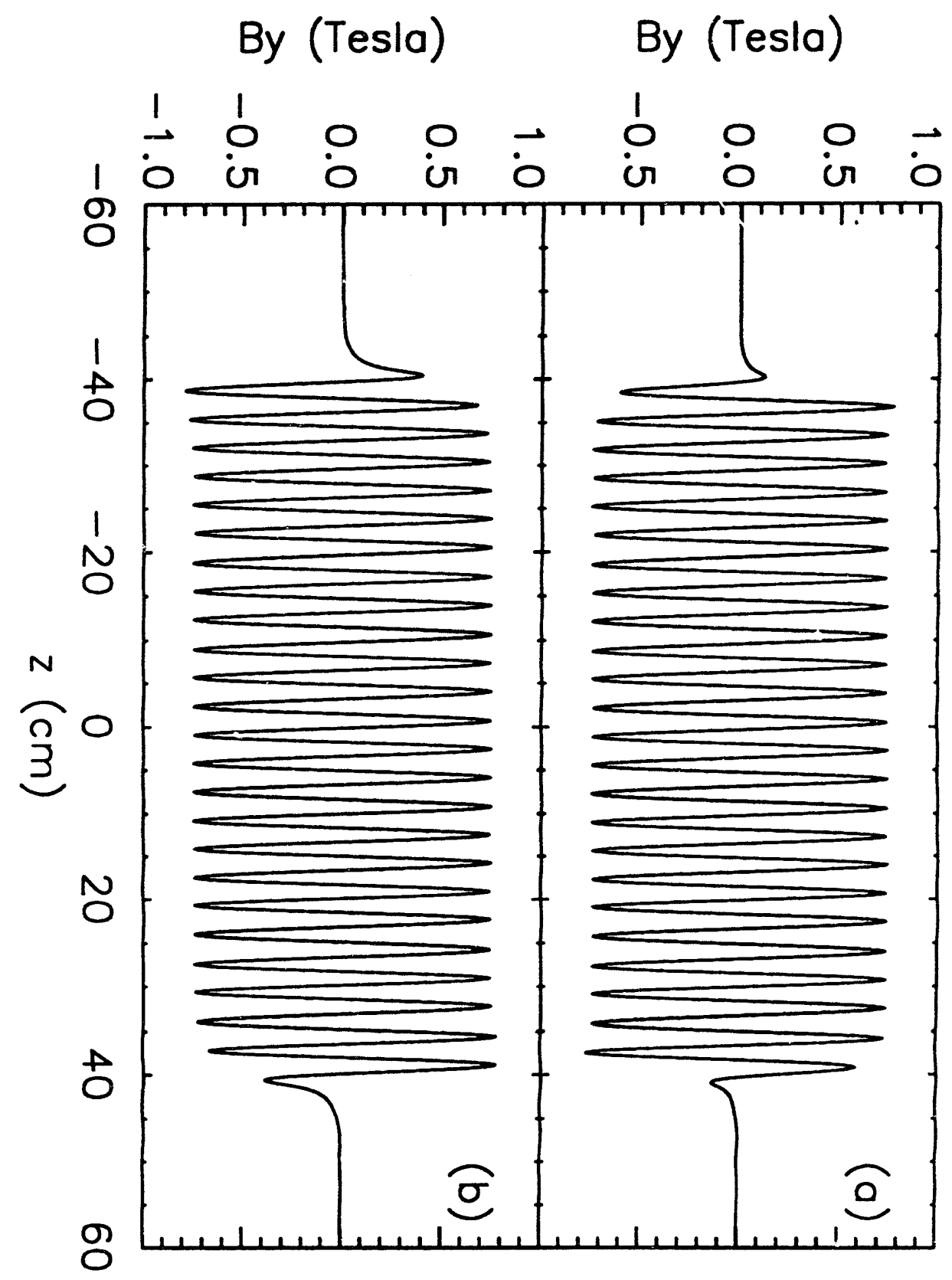




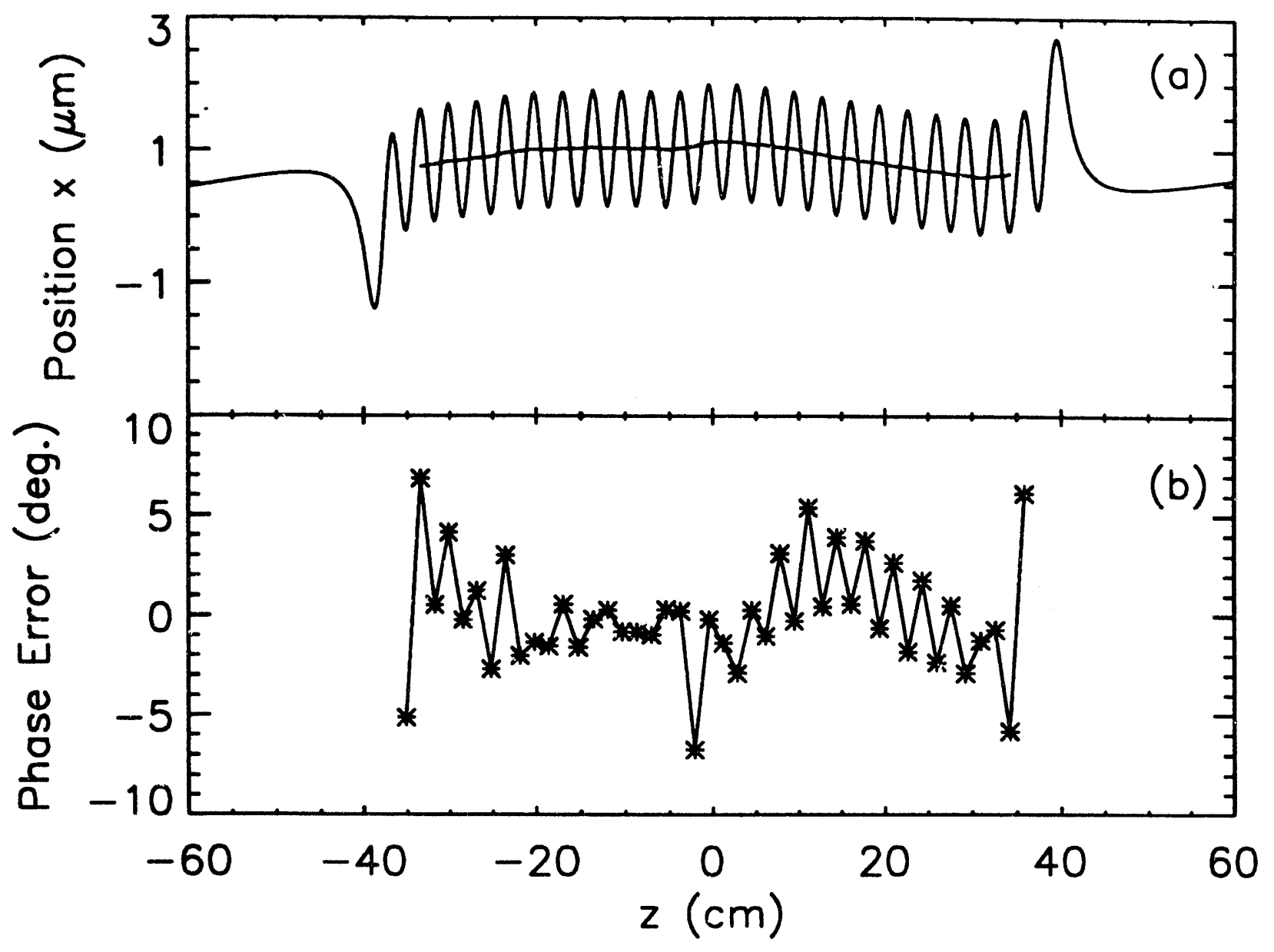

2. 


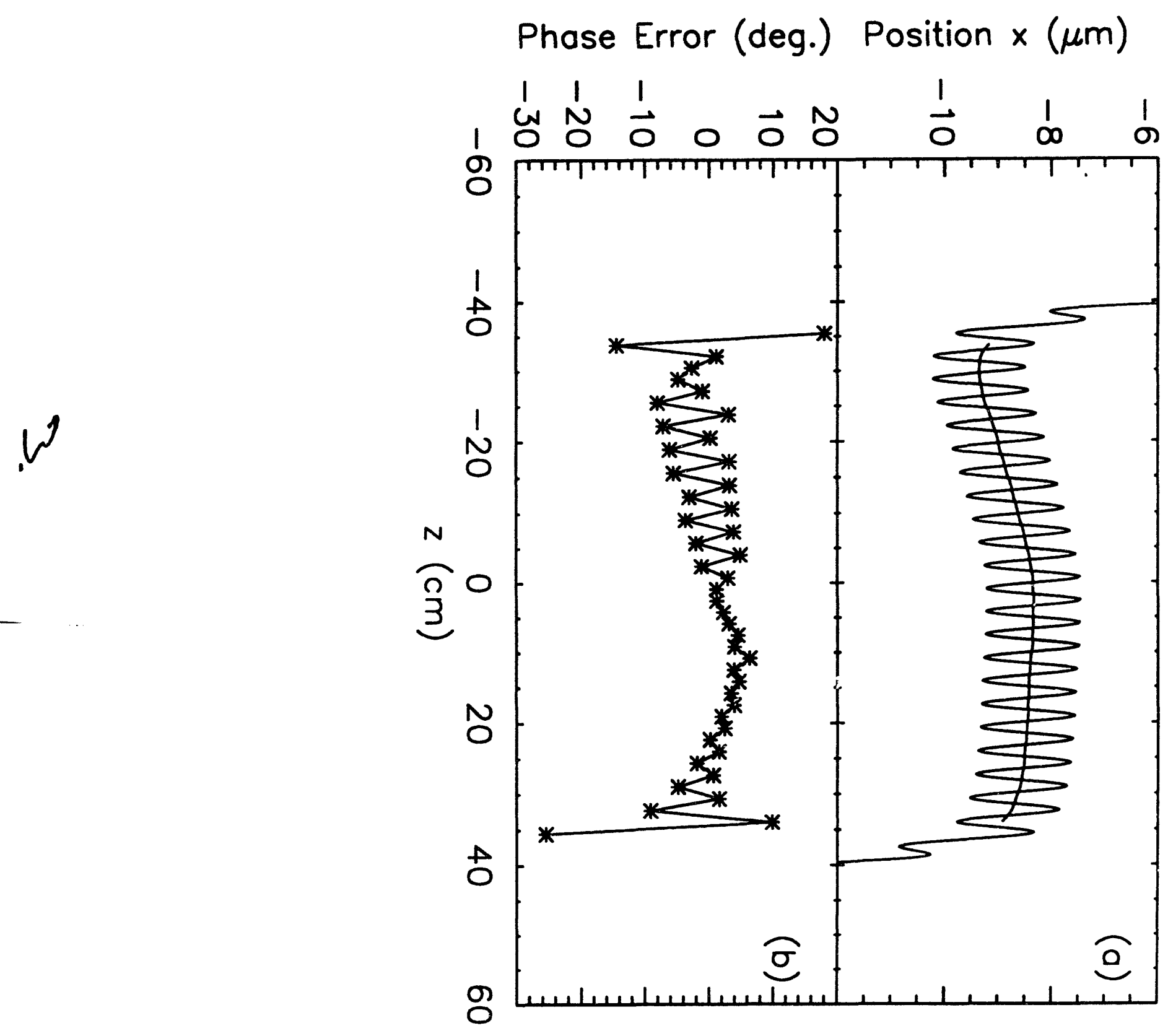




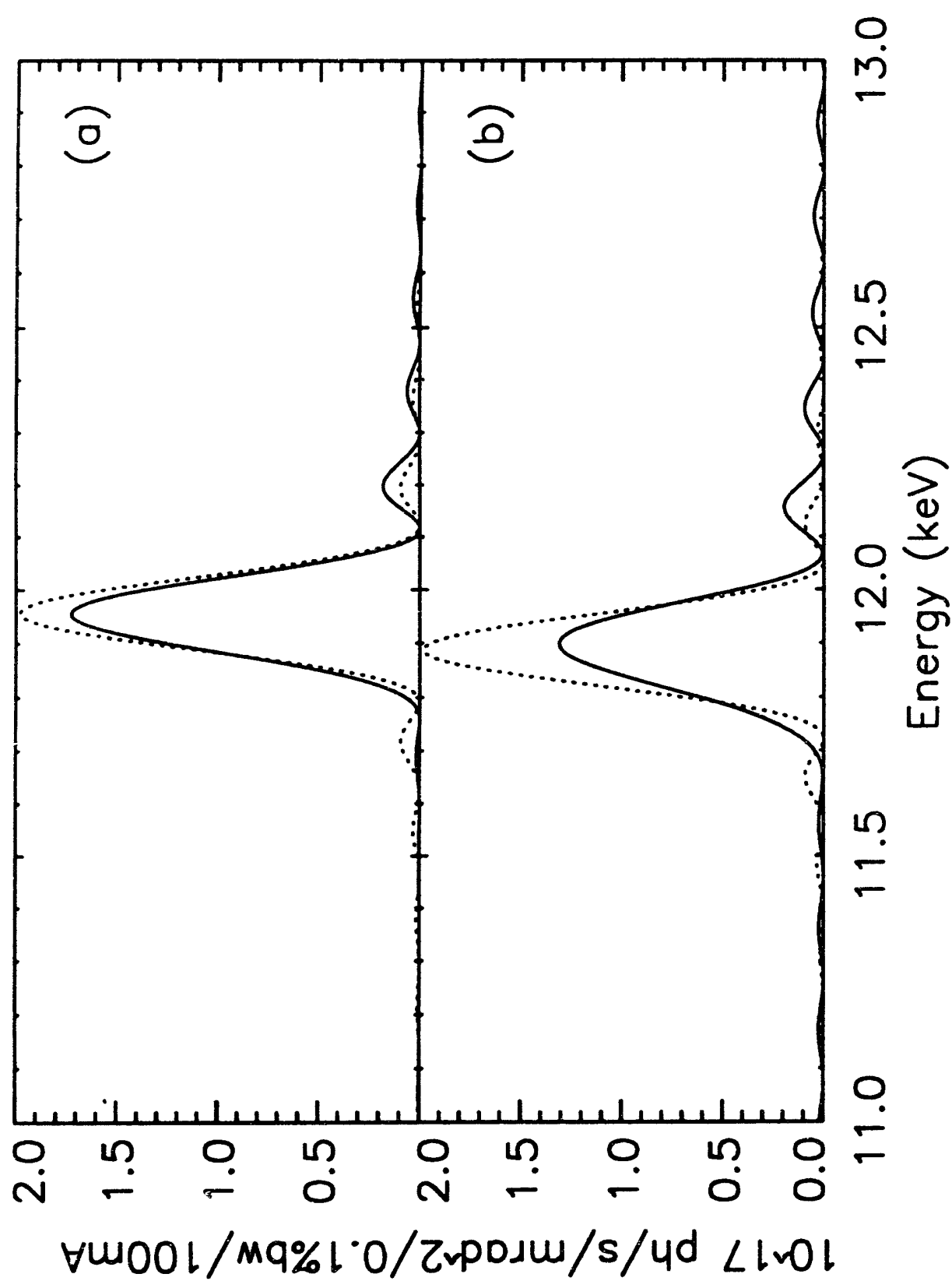

ז 

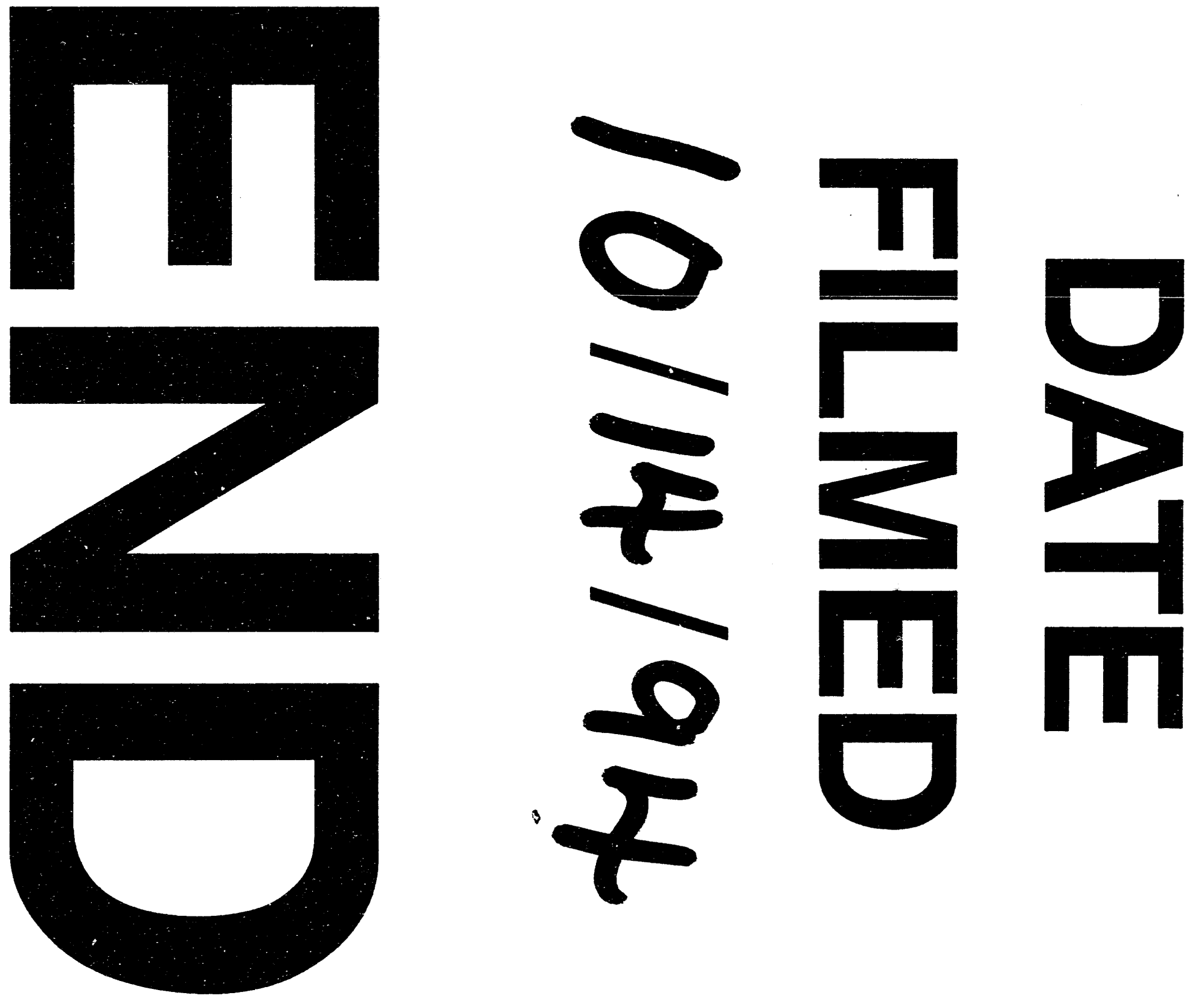

. 